\title{
DIRECTIONS OF EXTREME STIFFNESS AND STRENGTH IN LINEAR ELASTIC ANISOTROPIC SOLIDS
}

\author{
SUMMARY \\ An investigation for directions of extreme - maximum or minimum - values of the longitudinal and transverse stiffness \\ moduli as well as of the limit uniaxial and limit shear stresses in anisotropic linear elastic solids is performed in the pa- \\ per. The cases of cubic symmetry (regular crystal system) and of volumetrically isotropic cylindrical symmetry (hexago- \\ nal crystal system with additional constraints) are considered. The systems of non-linear equations for the components \\ of the versors of investigated directions are derived with use of the spectral decomposition of the elasticity (stiffness and \\ compliance) tensors.
}

Keywords: linear elasticity, anisotropy, stiffness moduli, strength, optimization, material design

\section{KIERUNKI EKSTREMALNEJ SZTYWNOŚCI I WYTRZYMALOŚCI W ANIZOTROPOWYCH CIAEACH LINIOWO SPRĘŻYSTYCH}

W pracy analizowany jest problem poszukiwania kierunków ekstremalnych - maksymalnych lub minimalnych - wartości modułów sztywności podlużnej i poprzecznej, jak również maksymalnego naprężenia normalnego $w$ stanie jednoosiowym i naprężenia stycznego w czystym ścinaniu w anizotropowych ciałach liniowo sprężystych dla przypadków symetrii kubicznej (regularny układ krystalograficzny) oraz objętościowo izotropowej symetrii cylindrycznej (heksagonalny układ krystalograficzny z dodatkowymi więzami). Wyprowadzono, przy użyciu rozkładu spektralnego tensorów sprężystości (sztywności i podatności), układ nieliniowych równań na składowe wersorów poszukiwanych kierunków.

Stowa kluczowe: liniowa sprężystość, anizotropia, moduly sztywności, state sprężyste, wytrzymałość, optymalizacja, inżynieria materiałowa

\section{List of nomenclature}

$$
\begin{gathered}
\mathrm{a}, \mathrm{b}, \ldots, \alpha, \beta-\text { scalars } \\
\mathbf{a}, \mathbf{b}, \ldots-\text { vectors } \\
\boldsymbol{\alpha}, \boldsymbol{\beta}, \ldots-2^{\text {nd }} \text { rank tesnors } \\
\mathbf{A}, \mathbf{B}, \ldots-4^{\text {th }} \text { rank tesnors } \\
\mathrm{x}_{1}, \mathrm{x}_{2}, \mathrm{x}_{2}-\text { Cartesian coordinates } \\
\boldsymbol{\alpha}-\text { stress tesnor } \\
\boldsymbol{\varepsilon}-\text { strain tesnor } \\
\mathbf{C}, \mathbf{S}-\text { elasticity tesnors }
\end{gathered}
$$

\section{MOTIVATION}

Linear elastic material remains still one of the most general and one of the most widely used constitutive model of deformable solid. It is also found useful in description of certain modern materials, particularly those exhibiting anisotropy of their mechanical properties e.g. crystalline or composite materials with controlled internal symmetry. The problems of description of anisotropic materials are far less extensively researched than it is in case of isotropy - as a result, the mathematical tools for designing and optimization of use of anisotropic materials may be still the subject of development. The current paper deals with the problem of finding such directions in the given linear elastic material, which are respective for the extreme values of the longitudinal and transverse stiffness moduli. It will be shown that this pro-
$\mathbf{H}$ - limit state tesnor

$\mathbf{P}_{\mathrm{K}}$ - orthogonal projector on $\mathrm{K}$-th eigensubspace

$\lambda_{\mathrm{K}}-\mathrm{K}^{\text {th }}$ eigenvalue of $\mathbf{S}$

$\chi_{\mathrm{K}}^{-2}-\mathrm{K}^{\text {th }}$ eigenvalue of $\mathbf{H}$

$\mathrm{E}(\mathbf{n})$ - Young modulus respective for direction given by $\mathbf{n}$

$\mathrm{G}(\mathbf{m}, \mathbf{n})$ - Kirchhoff respective for direction given by $\mathbf{m}$ and $\mathbf{n}$

$\mathrm{v}_{\mathrm{ij}}$ - Poisson's ratio

$\mathrm{k}, \mathrm{k}_{\mathrm{s}}, \ldots-$ limit stresses

blem is equivalent to the problem of finding the directions of extreme strength at uniaxial tension/compression and at pure shear when a generalized von Mises quadratic limit criterion for anisotropic solids is assumed. Similar analysis can be found in the paper by Ostrowska-Maciejewska and Rychlewski written in 2001, yet in their paper only cubic symmetry was considered - here also a specific case of transverse isotropy is analyzed. The analysis performed in the mentioned paper was based also more on the comparison of the form of certain tensors rather than on derivation of a system of equations enabling finding the investigated directions. Presented methodology and obtained results may be used in the process of material design or in construction element's shape design in order to optimize the use of its stiffness (or compliance) or its strength capacity.

* AGH University of Science and Technology, Faculty of Mechanical Engineering and Robotics, Department of Strength and Fatigue of Materials and Structures, Krakow, Poland 


\section{GENERALIZED STIFFNESS MODULI OF LINEAR ELASTIC ANISOTROPIC SOLIDS}

We are considering the generalized Hooke's law:

$$
\begin{aligned}
& \left\{\begin{array}{l}
\boldsymbol{\varepsilon}=\mathbf{C} \cdot \boldsymbol{\sigma} \\
\boldsymbol{\sigma}=\mathbf{S} \cdot \boldsymbol{\varepsilon} \\
\mathbf{C} \circ \mathbf{S}=\mathbf{S} \circ \mathbf{C}=\mathbf{I}_{\mathbf{S}}
\end{array} \Leftrightarrow\right. \\
& \Leftrightarrow\left\{\begin{array}{l}
\varepsilon_{\mathrm{ij}}=\mathrm{C}_{\mathrm{ijkl}} \cdot \sigma_{\mathrm{kl}} \\
\sigma_{\mathrm{ij}}=\mathrm{S}_{\mathrm{ijkl}} \cdot \varepsilon_{\mathrm{kl}} \\
\mathrm{C}_{\mathrm{ijk}} \mathrm{S}_{\mathrm{klmn}}=\mathrm{S}_{\mathrm{ijk} 1} \mathrm{C}_{\mathrm{klmn}}=\frac{1}{2}\left[\delta_{\mathrm{im}} \delta_{\mathrm{jn}}+\delta_{\mathrm{in}} \delta_{\mathrm{jm}}\right]
\end{array},\right.
\end{aligned}
$$

where $\boldsymbol{\sigma}$ is the Cauchy stress tensor, $\boldsymbol{\varepsilon}$ is the small strain tensor and $\mathbf{C}$ and $\mathbf{S}$ are fourth order compliance and stiffness tensors respectively, satisfying the following symmetry conditions:

$$
\begin{aligned}
\mathrm{S}_{\mathrm{ijkl}} & =\mathrm{S}_{\mathrm{jikl}}=\mathrm{S}_{\mathrm{ijlk}}=\mathrm{S}_{\mathrm{klij}} \\
\mathrm{C}_{\mathrm{ijkl}} & =\mathrm{C}_{\mathrm{jikl}}=\mathrm{C}_{\mathrm{ijlk}}=\mathrm{C}_{\mathrm{klij}}
\end{aligned}
$$

If the space of the stress states is considered as a dimensionless one (eg. as space of relative stresses), then both stress and strain state spaces can be considered as a single six-dimensional linear space of symmetric second order tensors. Then the elasticity tensors $\mathbf{C}$ and $\mathbf{S}$ act as a linear automorphic map in that space. For any such a map its eigenvalue problem can be stated, namely the problem of finding such a symmetric $2^{\text {nd }}$ order tensor $\omega$ and such a scalar $\lambda$ that:

$$
\mathbf{S} \cdot \omega=\lambda \omega .
$$

The solution of the eigenstate problem for the elasticity tensors leads to the spectral decomposition of those operators (Rychlewski 1984):

$$
\begin{aligned}
\mathbf{S} & =\lambda_{1} \mathbf{P}_{1}+\lambda_{2} \mathbf{P}_{2}+\ldots+\lambda_{\rho} \mathbf{P}_{\rho} \\
\mathbf{C} & =\frac{1}{\lambda_{1}} \mathbf{P}_{1}+\frac{1}{\lambda_{2}} \mathbf{P}_{2}+\ldots+\frac{1}{\lambda_{\rho}} \mathbf{P}_{\rho} \quad \rho \leq 6,
\end{aligned}
$$

where $\lambda_{K}(K=1, \ldots, \rho)$ are the eigenvalues of the stiffness tensor (so called Kelvin moduli), and $\mathbf{P}_{\mathrm{K}}(\mathrm{K}=1, \ldots, \rho)$ are the orthogonal projectors on the corresponding eigensubspaces. Detailed discussion on the spectral decomposition and specific properties of the eigenstates of elasticity tensors was a subject of numerous papers (e.g. Rychlewski 1984, Mehrabadi and Cowin 1990, Kowalczyk-Gajewska and Ostrowska-Maciejewska 2009).

In general, the Young modulus respective for a fixed direction given by a versor $\mathbf{n}$, being a proportionality coefficient between normal stress and linear strain along that direction, can be found through proper transformation (rotation) of the coordinate system and corresponding transformation of the components of the elasticity tensors:

$$
\mathrm{C}^{\prime}{ }_{\mathrm{ijkl}}=\alpha_{\mathrm{ip}} \alpha_{\mathrm{jq}} \alpha_{\mathrm{kr}} \alpha_{\mathrm{ls}} \mathrm{C}_{\mathrm{pqrs}} \text {, }
$$

where $\alpha_{i j}$ denote the components of the transformation matrix. If $\mathbf{n}$ coincide with the first coordinate axis of a new coordinate system then a new component $C^{\prime}{ }_{1111}$ denotes simply the inverse of the Young modulus corresponding with the direction of $\mathbf{n}$. In the same way one can find the value of the Kirchhoff modulus respective for two perpendicular directions given by versors $\mathbf{m}$ and $\mathbf{n}-$ if the first two axes of a new coordinate system are parallel to those directions, then $\mathrm{G}(\mathbf{m}, \mathbf{n})=\left[4 \mathrm{C}^{\prime}{ }_{1212}\right]^{-1}$. The above expressions are in general complex, however choosing as the basic coordinate system the one in which the coordinate axes coincide with the symmetry axes of the material simplifies them in great extent.

Spectral decomposition of the elasticity tensors enables simple expression of the elastic constants such as Young modulus, Kirchhoff modulus, bulk modulus or Poisson's ratio in case of anisotropic solids for any direction of stiffness examination in terms of the considered directions' versors and eigenvalues and orthogonal projectors of the elasticity tensors - quantities which are given uniquely for each considered material. In the current paper the longitudinal stiffness modulus (Young modulus) and transverse stiffness modulus (Kirchhoff modulus) are considered - they can be defined in the following way (Rychlewski 1984):

$$
\begin{aligned}
& \frac{1}{\mathrm{E}(\mathbf{n})}=(\mathbf{n} \otimes \mathbf{n}) \cdot \mathbf{C} \cdot(\mathbf{n} \otimes \mathbf{n})= \\
& =\frac{1}{\lambda_{1}}(\mathbf{n} \otimes \mathbf{n}) \cdot \mathbf{P}_{1} \cdot(\mathbf{n} \otimes \mathbf{n})+\ldots+ \\
& +\frac{1}{\lambda_{\rho}}(\mathbf{n} \otimes \mathbf{n}) \cdot \mathbf{P}_{\rho} \cdot(\mathbf{n} \otimes \mathbf{n}) \\
& \frac{1}{\mathrm{G}(\mathbf{n}, \mathbf{m})}=4(\mathbf{n} \otimes \mathbf{m}) \cdot \mathbf{C} \cdot(\mathbf{m} \otimes \mathbf{n})= \\
& =\frac{4}{\lambda_{1}}(\mathbf{n} \otimes \mathbf{m}) \cdot \mathbf{P}_{1} \cdot(\mathbf{m} \otimes \mathbf{n})+\ldots+ \\
& +\frac{4}{\lambda_{\rho}}(\mathbf{n} \otimes \mathbf{m}) \cdot \mathbf{P}_{\rho} \cdot(\mathbf{m} \otimes \mathbf{n}),
\end{aligned}
$$

where $\mathbf{n}$ and $\mathbf{m}$ are unitary vectors parallel to the directions of the stiffness examination such that $\mathbf{m} \cdot \mathbf{m}=\mathbf{n} \cdot \mathbf{n}=1$, $\mathbf{m} \cdot \mathbf{n}=0$. It is worth noting that the Young longitudinal modulus as well as the Kirchhoff shear modulus are inverse proportional to the elastic energy densities stored during the processes of uniaxial stretching/compression or pure shear respectively:

$$
\begin{aligned}
& \frac{1}{\mathrm{E}(\mathbf{n})}=2 \phi(\boldsymbol{\sigma}=(\mathbf{n} \otimes \mathbf{n})), \quad \frac{1}{2 \mathrm{G}(\mathbf{n}, \mathbf{m})}= \\
& =2 \phi(\boldsymbol{\sigma}=(\mathbf{n} \otimes \mathbf{m})+(\mathbf{m} \otimes \mathbf{n})), \\
& \text { where } \phi(\boldsymbol{\sigma})=\frac{1}{2} \boldsymbol{\sigma} \cdot \boldsymbol{\varepsilon}=\frac{1}{2} \boldsymbol{\sigma} \cdot \mathbf{C} \cdot \boldsymbol{\sigma} .
\end{aligned}
$$


This indicates that the directions of maximum (minimum) stiffness are the directions for which the stored elastic energy density is minimum (maximum) for fixed level of stress.

\section{GENERALIZED LIMIT CONDITION FOR ANISOTROPIC SOLIDS BY RICHARD VON MISES}

As it was mentioned in the beginning of the paper, the problem of finding the directions of extreme stiffness is equivalent to the problem of finding the directions of extreme value of a limit stress after assumption of a generalized quadratic limit condition for anisotropic solids, which was introduced by Mises in 1928, namely the limit condition of the following form (Mises 1928):

$$
\boldsymbol{\sigma} \cdot \mathbf{H} \cdot \boldsymbol{\sigma}=1,
$$

where $\mathbf{H}$ is the fourth rank limit state tensor satisfying the symmetry conditions (2). In case of uniaxial stress state $\boldsymbol{\sigma}=\sigma(\mathbf{n} \otimes \mathbf{n})$, the limit condition is satisfied for certain limit value $\sigma=k$ and the limit condition can be rewritten in the following form:

$$
\frac{1}{\mathrm{k}^{2}}=(\mathbf{n} \otimes \mathbf{n}) \cdot \mathbf{H} \cdot(\mathbf{n} \otimes \mathbf{n}) .
$$

which is analog to the expression for the Young modulus. In the same way, the limit condition at pure shear $\boldsymbol{\sigma}=\tau[(\mathbf{m} \otimes \mathbf{n})+(\mathbf{n} \otimes \mathbf{m})]$ reaching the limit value $\tau=\mathrm{k}_{\mathrm{s}}$ can be rewritten in the form analog to the expression for the Kirchhoff modulus:

$$
\frac{1}{\mathrm{k}_{\mathrm{s}}^{2}}=4(\mathbf{n} \otimes \mathbf{m}) \cdot \mathbf{H} \cdot(\mathbf{m} \otimes \mathbf{n}),
$$

Performing the same analysis as in case of investigation for the directions of extreme stiffness, however with use of the limit state tensor $\mathbf{H}$ instead of the compliance tensor $\mathbf{C}$, enables determination of directions of extreme strength let's note that the uniaxial limit stress is assumed to be the same at tension and at compression.

\section{NECESSARY CONDITIONS \\ FOR STATIONARY VALUE \\ OF QUADRATIC SCALAR-VALUED TENSOR FUNCTION OF VECTOR ARGUMENT}

In face of the above remarks, the most general statement of the analyzed problem is to find such unitary, mutually orthogonal three-dimensional Euclidean vectors $\mathbf{x}$ and $\mathbf{y}$ that the scalar-valued, quadratic tensor functions of a vector argument which are given by certain fourth order tensor A satisfying symmetry conditions (2), namely the functions

$$
\begin{aligned}
& f(\mathbf{x})=(\mathbf{x} \otimes \mathbf{x}) \cdot \mathbf{A} \cdot(\mathbf{x} \otimes \mathbf{x}) \\
& g(\mathbf{x}, \mathbf{y})=(\mathbf{x} \otimes \mathbf{y}) \cdot \mathbf{A} \cdot(\mathbf{y} \otimes \mathbf{x})
\end{aligned}
$$

reach stationary values. In order to formulate the necessary conditions for reaching the stationary value, we shall use the well-known Lagrange multipliers method of finding the function's extremum with assumption of additional constraints, namely:

$$
\begin{aligned}
& c_{1}(\mathbf{x})=\mathbf{x} \cdot \mathbf{x}-1=0 \\
& c_{2}(\mathbf{y})=\mathbf{y} \cdot \mathbf{y}-1=0 \\
& c_{3}(\mathbf{x} \cdot \mathbf{y})=\mathbf{x} \cdot \mathbf{y}=0
\end{aligned}
$$

We introduce functions:

$$
\begin{aligned}
& \varphi\left(\mathbf{x}, \mu_{1}\right)=f(\mathbf{x})+\mu_{1} \mathbf{c}_{1}(\mathbf{x}) \\
& \psi\left(\mathbf{x}, \mathbf{y}, v_{1}, v_{2}, v_{3}\right)=g(\mathbf{x}, \mathbf{y})+v_{1} \mathrm{c}_{1}(\mathbf{x})+ \\
& +v_{2} \mathbf{c}_{2}(\mathbf{y})+v_{3} \mathrm{c}_{3}(\mathbf{x}, \mathbf{y}),
\end{aligned}
$$

in which constant scalars $\mu_{1}, v_{1}, v_{2}, v_{3}$ are the Lagrange multipliers. The necessary conditions for the stationary values of $f$ and $g$ are given by the constraints (14) and the condition of vanishing of the linear part of the increment of value of $\varphi$ and $\psi$ respective for the infinitesimal increment of those functions' arguments. These conditions may be derived by setting those functions' Gâteaux derivatives equal zero.

First, let's consider the function of one variable $f$ and the correlated function $\varphi$ :

$$
\begin{array}{ll}
\partial_{\mathbf{x}} \varphi\left(\mathbf{x}, \mu_{1}\right)[\Delta \mathbf{x}]=\left.\frac{\mathrm{d}}{\mathrm{d} \alpha} \varphi\left(\mathbf{x}+\alpha \Delta \mathbf{x}, \mu_{1}\right)\right|_{\alpha=0} & = \\
=2\left[2(\mathbf{A} \cdot(\mathbf{x} \otimes \mathbf{x})) \cdot \mathbf{x}+\mu_{1} \mathbf{x}\right] \cdot \Delta \mathbf{x}=0 & \underset{\Delta \mathbf{x}}{\forall}
\end{array}
$$

We obtain then:

$$
2(\mathbf{A} \cdot(\mathbf{x} \otimes \mathbf{x})) \cdot \mathbf{x}=-\mu_{1} \mathbf{x}
$$

Multiplying it with $\mathbf{x}$ and using the first constraint $\mathbf{x} \cdot \mathbf{x}=1$, we can find the value of the multiplier $\mu_{1}$ and eliminate it from the condition's formulation:

$$
\mu_{1}=-2(\mathbf{x} \otimes \mathbf{x}) \cdot \mathbf{A} \cdot(\mathbf{x} \otimes \mathbf{x})
$$

The necessary condition for the stationary value of $f$ is then of the following form:

$$
(\mathbf{A} \cdot(\mathbf{x} \otimes \mathbf{x})) \cdot \mathbf{x}=[(\mathbf{x} \otimes \mathbf{x}) \cdot \mathbf{A} \cdot(\mathbf{x} \otimes \mathbf{x})] \mathbf{x}
$$

Cowin and Mehrabadi have shown that if the tensor A is the elasticity tensor, then the above condition is the necessary condition for the material to have a plane of symmetry of normal x (Cowin, Mehrabadi 1987).

In case of function of two variables $g$ and the correlated function $\psi$, we obtain:

$$
\begin{aligned}
& \partial_{\mathbf{x}} \psi\left(\mathbf{x}, \mathbf{y}, v_{1}, v_{2}, v_{3}\right)[\Delta \mathbf{x}]= \\
& =\left.\frac{\mathrm{d}}{\mathrm{d} \alpha} \psi\left(\mathbf{x}+\alpha \Delta \mathbf{x}, \mathbf{y}, v_{1}, v_{2}, v_{3}\right)\right|_{\alpha=0}= \\
& =\left[2(\mathbf{A} \cdot(\mathbf{y} \otimes \mathbf{x})) \cdot \mathbf{y}+2 v_{1} \mathbf{x}+v_{3} \mathbf{y}\right] \cdot \Delta \mathbf{x}=0 \quad \underset{\Delta \mathbf{x}}{\forall}
\end{aligned}
$$




$$
\begin{aligned}
& \partial_{\mathbf{y}} \psi\left(\mathbf{x}, \mathbf{y}, v_{1}, v_{2}, v_{3}\right)[\Delta \mathbf{y}]= \\
& =\left.\frac{\mathrm{d}}{\mathrm{d} \alpha} \psi\left(\mathbf{x}, \mathbf{y}+\alpha \Delta \mathbf{y}, v_{1}, v_{2}, v_{3}\right)\right|_{\alpha=0}= \\
& =\left[2(\mathbf{A} \cdot(\mathbf{x} \otimes \mathbf{y})) \cdot \mathbf{x}+2 v_{2} \mathbf{y}+v_{3} \mathbf{x}\right] \cdot \Delta \mathbf{y}=0 \quad \underset{\Delta \mathbf{y}}{\forall}
\end{aligned}
$$

We obtain then:

$$
\left\{\begin{array}{l}
2(\mathbf{A} \cdot(\mathbf{y} \otimes \mathbf{x})) \cdot \mathbf{y}+2 v_{1} \mathbf{x}+v_{3} \mathbf{y}=0 \\
2(\mathbf{A} \cdot(\mathbf{x} \otimes \mathbf{y})) \cdot \mathbf{x}+2 v_{2} \mathbf{y}+v_{3} \mathbf{x}=0
\end{array}\right.
$$

Multiplying the first equation with $\mathbf{x}$ and the second one with $\mathbf{y}$, after use of the constraints (14), we can find the values of the multipliers $v_{1}$ and $v_{2}$ and eliminate then from the condition's formulation:

$$
v_{1}=v_{2}=-(\mathbf{x} \otimes \mathbf{y}) \cdot \mathbf{A} \cdot(\mathbf{y} \otimes \mathbf{x})
$$

Substituting those relations to (22) and multiplying then the first equation with $\mathbf{y}$ and the second one with $\mathbf{x}$, after use of constraints (14), we can put the multiplier $v_{3}$ on the right hand side of each equation and then subtract one from another obtaining the final result:

$$
(\mathbf{x} \otimes \mathbf{x}) \cdot \mathbf{A} \cdot(\mathbf{x} \otimes \mathbf{y})=(\mathbf{y} \otimes \mathbf{y}) \cdot \mathbf{A} \cdot(\mathbf{x} \otimes \mathbf{y})
$$

\section{CUBIC SYMMETRY}

In the below considerations, following notation of any symmetric second order tensor a and fourth order tensor A satisfying symmetry conditions (2) will be used:

$$
\begin{aligned}
\mathbf{A} & =\left[\begin{array}{cccccc}
\mathrm{A}_{1111} & \mathrm{~A}_{1122} & \mathrm{~A}_{1133} & \sqrt{2} \mathrm{~A}_{1123} & \sqrt{2} \mathrm{~A}_{1131} & \sqrt{2} \mathrm{~A}_{1112} \\
& \mathrm{~A}_{2222} & \mathrm{~A}_{2233} & \sqrt{2} \mathrm{~A}_{2223} & \sqrt{2} \mathrm{~A}_{2231} & \sqrt{2} \mathrm{~A}_{2212} \\
& & \mathrm{~A}_{3333} & \sqrt{2} \mathrm{~A}_{3323} & \sqrt{2} \mathrm{~A}_{3331} & \sqrt{2} \mathrm{~A}_{3312} \\
& & & 2 \mathrm{~A}_{2323} & 2 \mathrm{~A}_{2331} & 2 \mathrm{~A}_{2312} \\
& \text { sym } & & 2 \mathrm{~A}_{3131} & 2 \mathrm{~A}_{3112} \\
& & & & 2 \mathrm{~A}_{1212}
\end{array}\right]_{\mathrm{S}} \\
\mathbf{a} & =\left[\begin{array}{c}
\mathrm{a}_{11} \\
\mathrm{a}_{22} \\
\mathrm{a}_{33} \\
\sqrt{2} \mathrm{a}_{23} \\
\sqrt{2} \mathrm{a}_{31} \\
\sqrt{2} \mathrm{a}_{12}
\end{array}\right]_{\mathrm{S}}
\end{aligned}
$$

Cubic symmetry, which is respective for the regular crystal system, is characterized by three pairwise orthogonal, mutually equivalent directions. We shall use the Cartesian coordinate system, in which the system axes are parallel to those specified directions and are given by versors $\mathbf{i}, \mathbf{j}, \mathbf{k}$. In such a coordinate system any tensor of cubic symmetry can be represented in the following general form:

$$
\mathbf{A}=\left[\begin{array}{cccccc}
\mathrm{a} & \mathrm{b} & \mathrm{b} & 0 & 0 & 0 \\
& \mathrm{a} & \mathrm{b} & 0 & 0 & 0 \\
& & \mathrm{a} & 0 & 0 & 0 \\
& & & \mathrm{c} & 0 & 0 \\
& \mathrm{sym} & & & \mathrm{c} & 0 \\
& & & & & \mathrm{c}
\end{array}\right]_{\mathrm{S}}
$$

The spectral decomposition of the tensor of cubic symmetry leads to the following result:

1) One-dimensional eigensubspace of spherical tensors

2) Two-dimensional eigensubspace of deviators

3) Three-dimensional eigensubspace of deviators

$$
\mathbf{A}=\mathrm{A}_{1} \mathbf{P}_{1}+\mathrm{A}_{2} \mathbf{P}_{2}+\mathrm{A}_{3} \mathbf{P}_{3}
$$

where the eigenvalues of $\mathbf{A}$ are equal:

$$
\mathrm{A}_{1}=\mathrm{a}+2 \mathrm{~b} \quad \mathrm{~A}_{2}=\mathrm{a}-\mathrm{b} \quad \mathrm{A}_{3}=\mathrm{c}
$$

and the orthogonal projectors:

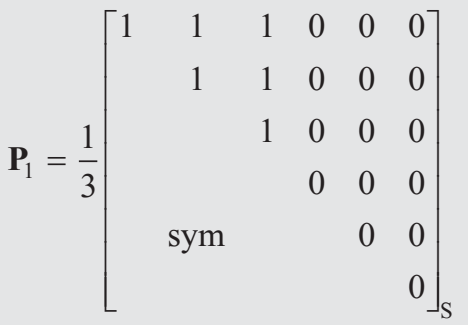

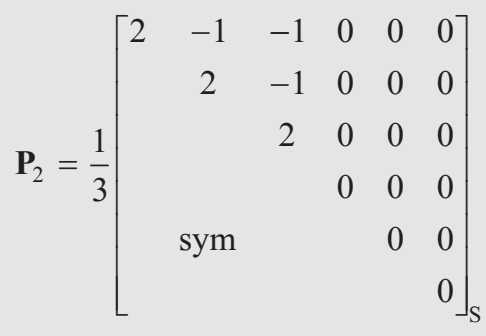

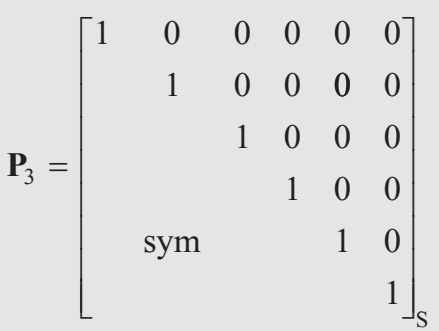

The spectral decomposition (27) may be rewritten in the following form:

$$
\mathbf{A}=\mathrm{C}_{1}(\mathbf{1} \otimes \mathbf{1})+\mathrm{C}_{2} \mathbf{K}+\mathrm{C}_{3} \mathbf{I}_{\mathrm{S}}
$$

where:

$$
\mathrm{C}_{1}=\frac{1}{3}\left(\mathrm{~A}_{1}-\mathrm{A}_{2}\right) \quad \mathrm{C}_{2}=\mathrm{A}_{2}-\mathrm{A}_{3} \quad \mathrm{C}_{3}=\mathrm{A}_{3}
$$




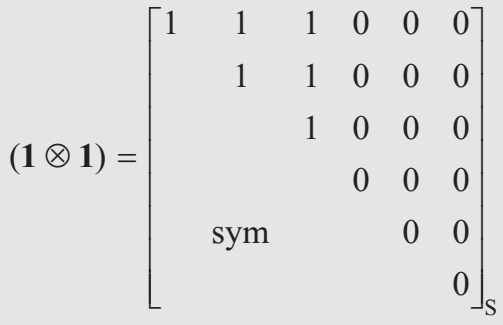

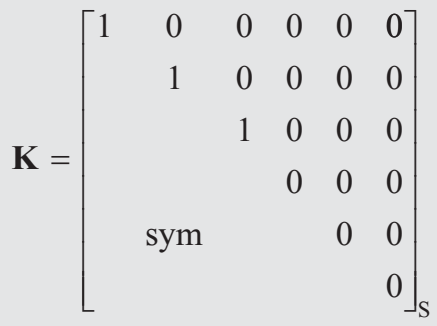

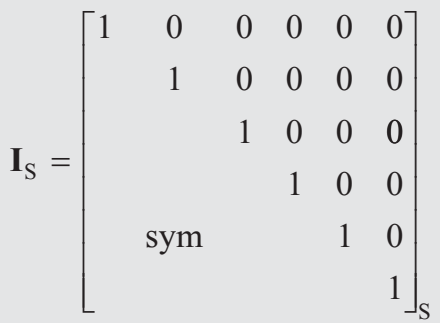

$\mathbf{1}$ is an identity second rank tensor, $\mathbf{I}_{\mathbf{S}}$ is an identity operator in the space of symmetric second order tensors, and $\mathbf{K}=\mathbf{i} \otimes \mathbf{i} \otimes \mathbf{i} \otimes \mathbf{i}+\mathbf{j} \otimes \mathbf{j} \otimes \mathbf{j} \otimes \mathbf{j}+\mathbf{k} \otimes \mathbf{k} \otimes \mathbf{k} \otimes \mathbf{k}$.

\subsection{Directions of extreme Young modulus and extreme uniaxial limit stress}

Substituting the decomposition (28) in the condition (20), we obtain:

$$
\begin{aligned}
& {\left[\left(\mathrm{C}_{1}(\mathbf{1} \otimes \mathbf{1})+\mathrm{C}_{2} \mathbf{K}+\mathrm{C}_{3} \mathbf{I}_{\mathrm{S}}\right) \cdot(\mathbf{x} \otimes \mathbf{x})\right] \cdot \mathbf{x}=} \\
& =\left[(\mathbf{x} \otimes \mathbf{x}) \cdot\left(\mathrm{C}_{1}(\mathbf{1} \otimes \mathbf{1})+\mathrm{C}_{2} \mathbf{K}+\mathrm{C}_{3} \mathbf{I}_{\mathrm{S}}\right) \cdot(\mathbf{x} \otimes \mathbf{x})\right] \mathbf{x}
\end{aligned}
$$

After proper multiplication the above vector equation can be written as a following system of three nonlinear equations involving three component of the vector $\mathbf{x}=\left[\begin{array}{lll}\mathrm{x}_{1} & \mathrm{x}_{2} & \mathrm{x}_{3}\end{array}\right]^{\mathrm{T}}$ :

$$
\left\{\begin{array}{l}
x_{1}^{2}=x_{1}^{4}+x_{2}^{4}+x_{3}^{4} \\
x_{2}^{2}=x_{1}^{4}+x_{2}^{4}+x_{3}^{4} \\
x_{3}^{2}=x_{1}^{4}+x_{2}^{4}+x_{3}^{4}
\end{array}\right.
$$

Let's note that the above equation does not depend on the eigenvalues of the tensor $\mathbf{A}$ - the directions of extreme values of function $f$ are independent on the components of the tensor $\mathbf{A}$ and are uniquely determined by the symmetry class of A. It is easy to find at least three solutions of the above system of equations:

- Principal directions of the regular system (e.g. [100] and other equivalent).

- Plane diagonal directions (e.g. [110] and other equivalent).

- Diagonal directions (e.g. [111] and other equivalent).
It can be shown however, that although the second solution satisfies the necessary condition (30), it does not satisfy sufficient condition - it is in fact a saddle point of the hypersurface given by function $f$.

If the considered investigated quantity is the Young modulus and the tensor $\mathbf{A}$ is the compliance tensor $\mathbf{C}$, then its eigenvalues are equal:

$$
\frac{1}{\lambda_{1}}=\frac{1-2 v}{\mathrm{E}} \quad \frac{1}{\lambda_{2}}=\frac{1+v}{\mathrm{E}} \quad \frac{1}{\lambda_{3}}=\frac{1}{2 \mathrm{G}}
$$

where $\mathrm{E}$ is the Young modulus along the principal axes of symmetry, $v$ is the Poisson's ratio at tension along those directions and $\mathrm{G}$ is the Kirchhoff modulus when shearing in planes perpendicular to the principal symmetry axes along the other two axes. The values of the Young modulus corresponding to the directions found as a solution of (30) are equal:

- Longitudinal stiffness along principal axes of symmetry:

$$
\mathrm{E}_{[100]}=\mathrm{E}=\frac{3 \lambda_{1} \lambda_{2}}{2 \lambda_{1}+\lambda_{2}} \text {. }
$$

- Longitudinal stiffness along plane diagonals:

$$
E_{[110]}=\frac{4 E G}{E+2 G(1-v)}=\frac{6 \lambda_{1} \lambda_{2} \lambda_{3}}{3 \lambda_{1} \lambda_{2}+2 \lambda_{2} \lambda_{3}+\lambda_{1} \lambda_{3}} .
$$

- Longitudinal stiffness along spatial diagonals:

$$
E_{[111]}=\frac{3 E G}{E+G(1-2 v)}=\frac{3 \lambda_{1} \lambda_{3}}{2 \lambda_{1}+\lambda_{3}} \text {. }
$$

Although $E_{[110]}$ is not a stationary value of $E$ it may be still considered as a local extreme value of the Young modulus if only a special class of load is allowed, e.g. uniaxial stress in planes containing faces of the cubic cell (e.g. (100) plane and other equivalent $-E$ reaches local maximum then) or in planes containing both plane and spatial diagonals of the cells (e.g. (110) plane and other equivalent $-\mathrm{E}$ reaches local minimum then).

If the considered investigated quantity is the limit uniaxial stress in case of the generalized von Mises yield criterion for anisotropic solids and the tensor $\mathbf{A}$ is the limit state tensor $\mathbf{H}$, then - if pressure insensitivity is assumed according to the postulates of Mises (Mises 1928) - its eigenvalues are equal:

$$
\frac{1}{\chi_{1}^{2}}=0 \quad \frac{1}{\chi_{2}^{2}}=\frac{3}{2 \mathrm{k}^{2}}=\frac{1}{2 \mathrm{k}_{\mathrm{s} 45}^{2}} \quad \frac{1}{\chi_{3}^{2}}=\frac{1}{2 \mathrm{k}_{\mathrm{s}}^{2}}
$$

where $\chi_{1}=\infty$ is the limit hydrostatic stress, $\mathrm{k}$ is the limit uniaxial stress along the principal symmetry axes, $\mathrm{k}_{\mathrm{s} 45}^{2}$ is the limit shear stress when shearing in [110][1 $\overline{1} 0]$ directions (and equivalent) and $k_{s}$ is the limit shear stress when shearing in [100][010] directions (and equivalent). The extreme values of the limit uniaxial stress are equal:

- Tensile/compressive strength along principal axes of symmetry: $\mathrm{k}_{[100]}=\mathrm{k}$.

- Tensile/compressive strength along plane diagonals:

$$
\mathrm{k}_{[110]}=\sqrt{\frac{4 \mathrm{k}^{2} \mathrm{k}_{\mathrm{s}}^{2}}{\mathrm{k}^{2}+\mathrm{k}_{\mathrm{s}}^{2}}} .
$$

- Tensile/compressive strength along spatial diagonals:

$$
\mathrm{k}_{[111]}=\sqrt{3} \mathrm{k}_{\mathrm{s}} \text {. }
$$


a)

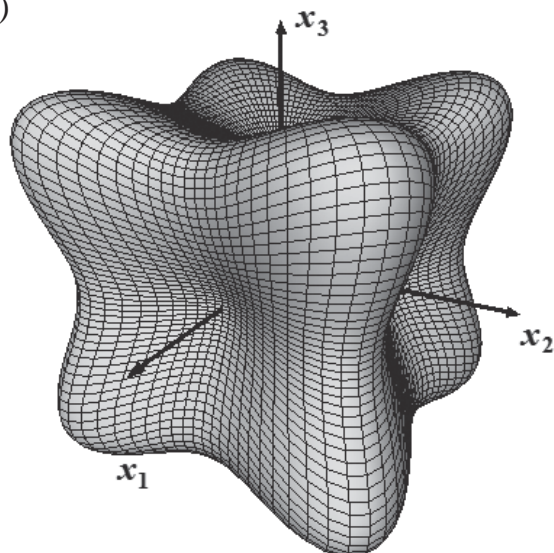

b)

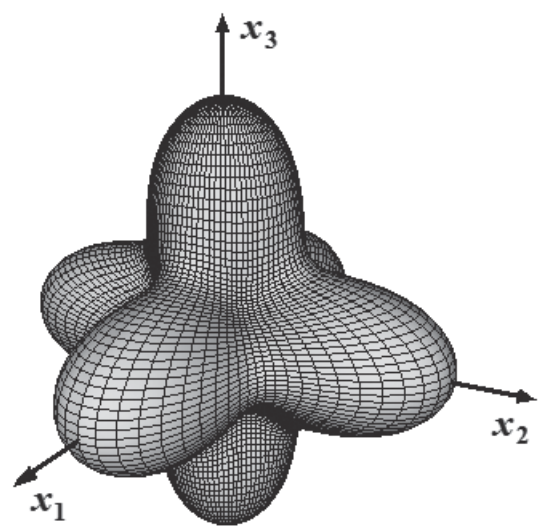

Fig. 1. Distribution of the Young modulus of copper depending on the direction of its examination (a); b) stored elastic energy density at uniaxial tension depending on the direction of loading

The surface determining the dependency of the Young modulus on the direction of for cubic symmetry is shown in the Figure $1 \mathrm{a}$ - the axes of the coordinate system coincide with the principal symmetry axes of regular cell. In the Figure $1 \mathrm{~b}$ stored elastic energy density at uniaxial stress state in various directions is presented. Experimental data used for the plots (Berryman 2005) are given below the figures. The general character of the stiffness distribution is rather easily predictable as it should correspond with the distances between neighboring atoms in a regular cell depending on the chosen direction.

Elastic constants of copper (Berryman 2005):

$$
\begin{array}{ll}
\mathrm{S}_{1111}=171,0 \mathrm{GPa} & \lambda_{1}=415,0 \mathrm{GPa}, \\
\mathrm{S}_{1122}=122,0 \mathrm{GPa} & \lambda_{2}=49,0 \mathrm{GPa}, \\
\mathrm{S}_{1212}=69,1 \mathrm{GPa} & \lambda_{3}=138,2 \mathrm{GPa} .
\end{array}
$$

\subsection{Directions of extreme kirchhoff modulus and extreme shear stress}

Substituting the decomposition (28) in the condition (24), after proper multiplication the nonlinear scalar equation of the condition (24) can be rewritten in terms of the components of the investigated vectors $\mathbf{x}$ and $\mathbf{y}$ in the following form:

$$
\begin{aligned}
& \left(x_{1}^{2}-y_{1}^{2}\right) \cdot\left(x_{1} y_{1}\right)+\left(x_{2}^{2}-y_{2}^{2}\right) \cdot\left(x_{2} y_{2}\right)+ \\
& +\left(x_{3}^{2}-y_{3}^{2}\right) \cdot\left(x_{3} y_{3}\right)=0
\end{aligned}
$$

It is easy to find two solutions of the equations:

- Principal directions of the regular system (e.g. [100][010] and other equivalent).

- Plane diagonal directions (e.g. $[110][1 \overline{1} 0]$ and other equivalent).

The values of the Kirchhoff modulus corresponding with those directions of shearing are equal:

- Principal directions of the regular system

$$
\mathrm{G}_{[100][010]}=\frac{\lambda_{3}}{2}=\mathrm{G} \text {. }
$$

- Plane diagonal directions

$$
\mathrm{G}_{[110][1 \overline{1} 0]}=\frac{\lambda_{2}}{2}=\frac{E}{2(1+v)} \text {. }
$$

The values of the limit shear stress corresponding with those directions of shearing are equal:

- Principal directions of the regular system

$$
\mathrm{k}_{\mathrm{s}[100][010]}=\mathrm{k}_{\mathrm{s}} \text {. }
$$

- Plane diagonal directions

$$
\mathrm{k}_{\mathrm{s}[110][1 \overline{1} 0]}=\mathrm{k}_{\mathrm{s} 45} \text {. }
$$

These are only single solutions satisfying the necessary condition for the directions given by $\mathbf{x}$ and $\mathbf{y}$ to be the directions of stationary values of proper functions. More detailed - yet still very general - discussion on the solution of that problem can be found in (Ostrowska-Maciejewska, Rychlewski 2001). Since the function $g(\mathbf{x}, \mathbf{y})$ is a function of three independent parameters (six components of two vectors constrained with two normalization conditions and one orthogonality condition), any visualization of that function would be rather complex and unclear.

\section{VOLUMETRICALLY ISOTROPIC CYLINDRICAL SYMMETRY}

Any fourth rank tensor satisfying symmetry conditions (2) such that one of its eigenstates is an isotropic second order tensor is termed to be volumetrically isotropic. Volumetrically isotropic elasticity tensors were subject of an analysis performed by Burzyński (Burzyński 1928) - for any material that can be described with such tensor, the decomposition of the elastic energy density into volumetric and distortional part is possible. Burzyński has given a set of relations which must be fulfilled by the components of a tensor, so that it was volumetrically isotropic. In case of the limit state tensors of the Mises' limit condition the volume isotropy is a consequence of the postulate of pressure insensitivity stated by Mises himself. Any tensor of volumetrically isotro- 
pic cylindrical symmetry with its symmetry axis coinciding with the third axis of the considered coordinate system can be represented in the following general form:

$$
\mathbf{A}=\left[\begin{array}{cccccc}
\mathrm{K}-(\mathrm{b}+\mathrm{c}) & \mathrm{b} & \mathrm{c} & 0 & 0 & 0 \\
& \mathrm{~K}-(\mathrm{b}+\mathrm{c}) & \mathrm{c} & 0 & 0 & 0 \\
& & \mathrm{~K}-2 \mathrm{c} & 0 & 0 & 0 \\
& & & \mathrm{~d} & 0 & 0 \\
& \mathrm{sym} & & & \mathrm{d} & 0 \\
& & & & & \mathrm{~K}-2 \mathrm{~b}-\mathrm{c}
\end{array}\right]_{\mathrm{S}}
$$

The spectral decomposition of the tensor of volumetrically isotropic cylindrical symmetry leads to the following result:

1) One-dimensional eigensubspace of spherical tensors.

2) One-dimensional eigensubspace of deviators.

3) Two two-dimensional eigensubspaces of deviators.

$$
\mathbf{A}=\mathrm{A}_{1} \mathbf{P}_{1}+\mathrm{A}_{2} \mathbf{P}_{2}+\mathrm{A}_{3} \mathbf{P}_{3}+\mathrm{A}_{4} \mathbf{P}_{4}
$$

where the eigenvalues of $\mathbf{A}$ are equal:

$$
\mathrm{A}_{1}=\mathrm{K}, \quad \mathrm{A}_{2}=\mathrm{K}-3 \mathrm{c}, \quad \mathrm{A}_{3}=\mathrm{K}-2 \mathrm{~b}-\mathrm{c}, \quad \mathrm{A}_{4}=\mathrm{d}
$$

and the orthogonal projectors:

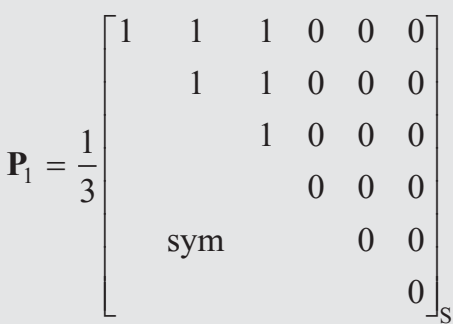

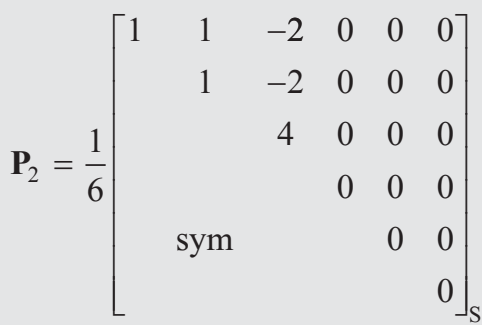

$$
\mathbf{P}_{3}=\frac{1}{2}\left[\begin{array}{cccccc}
1 & -1 & 0 & 0 & 0 & 0 \\
& 1 & 0 & 0 & 0 & 0 \\
& & 0 & 0 & 0 & 0 \\
& & & 0 & 0 & 0 \\
& \text { sym } & & & 0 & 0 \\
& & & & & 2
\end{array}\right]_{S}
$$

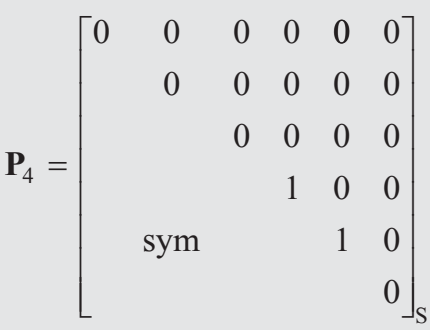

\subsection{Directions of extreme Young modulus and extreme uniaxial limit stress}

Due to high symmetry of the considered tensor, the whole problem may be simplified - knowing that the solution has to be axis-symmetric one of the in-plane components of the investigated versor may be chosen arbitrary. Let's assume the argument vector $\mathbf{x}$ in the following form $\mathbf{x}=\left[\begin{array}{lll}\sin \psi & 0 & \cos \psi\end{array}\right]^{\mathrm{T}}$, where $\psi \in\left(0,90^{\circ}\right)$ denotes the angle between the direction of uniaxial load and the direction of the specified symmetry axis. Substituting the decomposition (33) into the condition (20), after proper multiplication we obtain the following system of two nonlinear equations for the components of the versor of the investigated direction:

$$
\left\{\begin{array}{l}
\sin \psi\left[2 \mathrm{~A}_{1}+\mathrm{A}_{2}\left(1-3 \cos ^{2} \psi\right)+3 \mathrm{~A}_{3} \sin ^{2} \Psi+6 \mathrm{~A}_{4} \cos ^{2} \psi\right]= \\
=\sin \psi\left[2 \mathrm{~A}_{1}+\mathrm{A}_{2}\left(1-3 \cos ^{2} \psi\right)^{2}+3 \mathrm{~A}_{3} \sin ^{4} \psi+\right. \\
\left.+12 \mathrm{~A}_{4} \cos ^{2} \psi \sin ^{2} \psi\right] \\
\cos \psi\left[2 \mathrm{~A}_{1}+2 \mathrm{~A}_{2}\left(3 \cos ^{2} \psi-1\right)+6 \mathrm{~A}_{4} \sin ^{2} \psi\right]= \\
=\cos \psi\left[2 \mathrm{~A}_{1}+\mathrm{A}_{2}\left(3 \cos ^{2} \psi-1\right)^{2}+3 \mathrm{~A}_{3} \sin ^{4} \psi+\right. \\
\left.+12 \mathrm{~A}_{4} \cos ^{2} \psi \sin ^{2} \psi\right]
\end{array}\right.
$$

It can be shown that the above equations are fulfilled in case of $\psi=0^{\circ}$ (uniaxial load in the direction perpendicular to the specified symmetry axis) and $\psi=90^{\circ}$ (uniaxial load along the specified symmetry axis). Having checked those two cases it is now possible to divide the first equation by $\sin \psi$ and the second one by $\cos \psi-$ after proper transformation, both equations can be rewritten in the same following form

$$
\begin{aligned}
& \mathrm{A}_{2}\left(1-3 \cos ^{2} \psi\right)+\mathrm{A}_{3}\left(1-\cos ^{2} \psi\right)- \\
& -2 \mathrm{~A}_{4}\left(1-2 \cos ^{2} \psi\right)=0
\end{aligned}
$$

what yields in:

$$
\psi_{\mathrm{e}}=\operatorname{arctg} \sqrt{\frac{2\left(\mathrm{~A}_{2}-\mathrm{A}_{4}\right)}{\mathrm{A}_{2}+\mathrm{A}_{3}-2 \mathrm{~A}_{4}}}
$$

if only such root exists. If the considered investigated quantity is the Young modulus and the tensor $\mathbf{A}$ is the compliance tensor $\mathbf{C}$, then its eigenvalues are equal:

$$
\begin{aligned}
& \frac{1}{\lambda_{1}}=\frac{1-2 v_{\mathrm{LT}}}{\mathrm{E}_{\mathrm{L}}}=\frac{1-v_{\mathrm{TT}}-v_{\mathrm{TL}}}{\mathrm{E}_{\mathrm{T}}}, \\
& \frac{1}{\lambda_{2}}=\frac{1+v_{\mathrm{LT}}}{\mathrm{E}_{\mathrm{L}}}=\frac{1-v_{\mathrm{TT}}+2 v_{\mathrm{TL}}}{\mathrm{E}_{\mathrm{T}}}, \\
& \frac{1}{\lambda_{3}}=\frac{1}{2 \mathrm{G}_{\mathrm{T}}}, \quad \frac{1}{\lambda_{4}}=\frac{1}{2 \mathrm{G}_{\mathrm{LT}}},
\end{aligned}
$$

where $\mathrm{E}_{\mathrm{L}}$ and $\mathrm{E}_{\mathrm{T}}$ are the Young moduli along and perpendicularly to the specified symmetry axis respectively, $G_{L T}$ is the Kirchhoff modulus when shearing in plane containing the specified axis and $G_{T}$ is the Kirchhoff modulus when shearing in plane perpendicular to it; in the Poisson's ratios 
the first subscript indicate the direction of uniaxial load and the second one, the direction of transverse strain. The values of the Young modulus corresponding to the directions found as a solution of (35) are equal:

- Longitudinal stiffness along the specified axis of symmetry: $\mathrm{E}_{0^{\circ}}=\mathrm{E}_{\mathrm{L}}$.

- Longitudinal stiffness perpendicularly to the specified axis: $\mathrm{E}_{90^{\circ}}=\mathrm{E}_{\mathrm{T}}$.

The value of the Young modulus respective for the direction given by angle $\psi_{\mathrm{e}}$ depends on the components of the compliance tensor in a very complex way - it can be however easily calculated numerically. The angle $\psi_{\mathrm{e}}$ can be expressed in the following way:

$$
\begin{aligned}
& \psi_{\mathrm{e}}=\operatorname{arctg} \sqrt{\frac{2 \lambda_{3}\left(\lambda_{4}-\lambda_{2}\right)}{\lambda_{3} \lambda_{4}+\lambda_{2} \lambda_{4}-2 \lambda_{2} \lambda_{3}}}= \\
& =\operatorname{arctg} \sqrt{\frac{2 \mathrm{G}_{\mathrm{T}}\left[2 \mathrm{G}_{\mathrm{LT}}\left(1+\mathrm{v}_{\mathrm{LT}}\right)-\mathrm{E}_{\mathrm{L}}\right]}{2 \mathrm{G}_{\mathrm{T}} \mathrm{G}_{\mathrm{LT}}\left(1+\mathrm{v}_{\mathrm{LT}}\right)+\mathrm{E}_{\mathrm{L}} \mathrm{G}_{\mathrm{LT}}-2 \mathrm{E}_{\mathrm{L}} \mathrm{G}_{\mathrm{T}}}}
\end{aligned}
$$

If the considered investigated quantity is the limit uniaxial stress in case of the generalized von Mises yield criterion for anisotropic solids and the tensor $\mathbf{A}$ is the limit state tensor $\mathbf{H}$, then its eigenvalues are equal:

$$
\begin{aligned}
\frac{1}{\chi_{1}^{2}} & =0, \frac{1}{\chi_{2}^{2}}=\frac{3}{2 \mathrm{k}_{\mathrm{L}}^{2}}, \frac{1}{\chi_{3}^{2}}=\frac{1}{2 \mathrm{k}_{\mathrm{sT}}^{2}}=\frac{4 \mathrm{k}_{\mathrm{L}}^{2}-\mathrm{k}_{\mathrm{T}}^{2}}{2 \mathrm{k}_{\mathrm{L}}^{2} \mathrm{k}_{\mathrm{T}}^{2}}, \\
\frac{1}{\chi_{4}^{2}} & =\frac{1}{2 \mathrm{k}_{\mathrm{sLT}}^{2}}
\end{aligned}
$$

where $\chi_{1}=\infty$ is the limit hydrostatic stress, $\mathrm{k}_{\mathrm{L}}$ and $\mathrm{k}_{\mathrm{T}}$ denote limit uniaxial stress at tension or compression along and perpendicularly to the specified axis respectively, while $\mathrm{k}_{\mathrm{sLT}}$ and $\mathrm{k}_{\mathrm{sT}}$ denote limit shear stress when shearing in planes containing the specified axis or being perpendicular to it respectively. The angle determining the direction of extreme strength can be found after obtaining $\mathrm{k}_{\mathrm{L}}, \mathrm{k}_{\mathrm{T}}, \mathrm{k}_{\mathrm{sLT}}$ and $\mathrm{k}_{\mathrm{sT}}$ in simple strength tests:

$$
\begin{aligned}
& \psi_{\mathrm{s}}=\operatorname{arctg} \sqrt{\frac{2 \chi_{3}^{2}\left(\chi_{4}^{2}-\chi_{2}^{2}\right)}{\chi_{3}^{2} \chi_{4}^{2}+\chi_{2}^{2} \chi_{4}^{2}-2 \chi_{2}^{2} \chi_{3}^{2}}}= \\
& =\operatorname{arctg} \sqrt{\frac{2 \mathrm{k}_{\mathrm{sT}}^{2}\left(3 \mathrm{k}_{\mathrm{sLT}}^{2}-\mathrm{k}_{\mathrm{L}}^{2}\right)}{3 \mathrm{k}_{\mathrm{sT}}^{2} \mathrm{k}_{\mathrm{sLT}}^{2}+\mathrm{k}_{\mathrm{L}}^{2} \mathrm{k}_{\mathrm{sLT}}^{2}-2 \mathrm{k}_{\mathrm{L}}^{2} \mathrm{k}_{\mathrm{sT}}^{2}}}
\end{aligned}
$$

The surface determining the dependency of the Young modulus on the direction of examination is shown in the Figures $2 \mathrm{a}$ and $3 \mathrm{a}-$ the $\mathrm{x}_{1}$ axis of the coordinate system coincide with the principal symmetry axis of material. In the Figures $2 b$ and $3 b$ stored elastic energy density at uniaxial stress state in various directions is presented. Experimental data used for the plots are given below the figures (Berryman 2005, Yoo and Fu 1998). They refer to magnesium and titanium exhibiting hexagonal structure which in terms of continuum mechanics is respective for the cylindrical symmetry - furthermore, the data indicate that the considered materials are close to be volumetrically isotropic (Kowalczyk-Gajewska and Ostrowska-Maciejewska 2009).

Elastic constants of magnesium (Berryman 2005):

$$
\begin{array}{ll}
\mathrm{S}_{1111}=61,5 \mathrm{GPa}, & \lambda_{1}=415,0 \mathrm{GPa}, \\
\mathrm{S}_{1122}=21,4 \mathrm{GPa}, & \lambda_{2}=49,0 \mathrm{GPa}, \\
\mathrm{S}_{1212}=16,4 \mathrm{GPa}, & \lambda_{3}=138,2 \mathrm{GPa}, \\
\mathrm{S}_{2222}=59,3 \mathrm{GPa}, & \lambda_{4}=138,2 \mathrm{GPa} . \\
\mathrm{S}_{2233}=25,7 \mathrm{GPa}, &
\end{array}
$$

Elastic constants of titanium (Yoo and Fu 1998):

$$
\begin{array}{ll}
\mathrm{S}_{1111}=181,6 \mathrm{GPa}, & \lambda_{1}=105,7 \mathrm{GPa}, \\
\mathrm{S}_{1122}=68,9 \mathrm{GPa}, & \lambda_{2}=40,8 \mathrm{GPa}, \\
\mathrm{S}_{1212}=47,2 \mathrm{GPa}, & \lambda_{3}=33,6 \mathrm{GPa}, \\
\mathrm{S}_{2222}=163,9 \mathrm{GPa}, & \lambda_{4}=32,8 \mathrm{GPa} . \\
\mathrm{S}_{2233}=91,3 \mathrm{GPa}, &
\end{array}
$$

a)

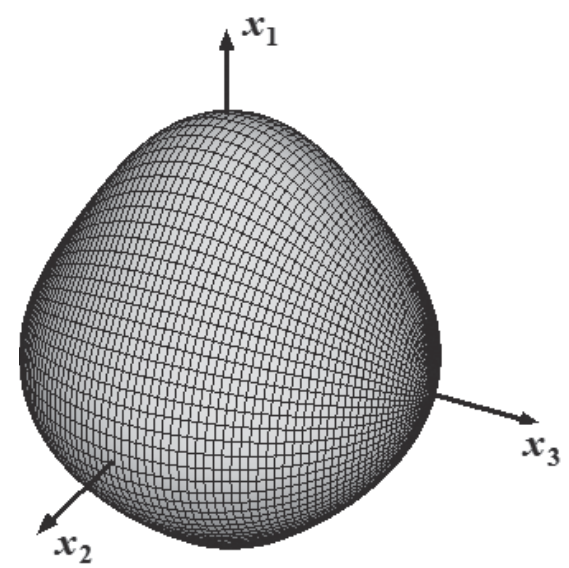

b)

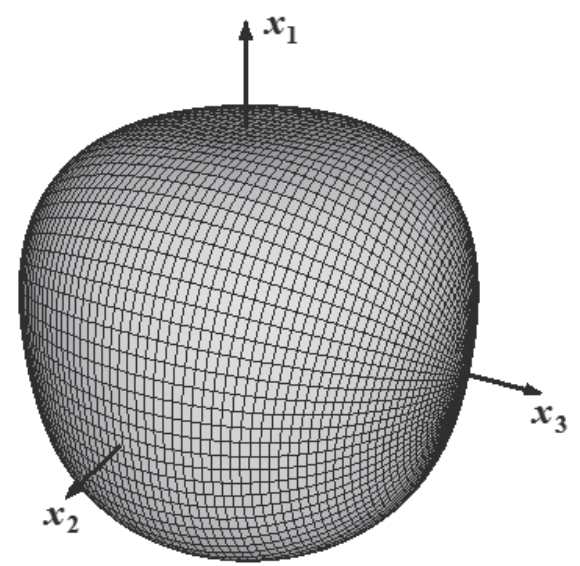

Fig. 2. Distribution of the Young modulus of magnesium depending on the direction of its examination (a); b) stored elastic energy density at uniaxial tension depending on the direction of loading 
a)

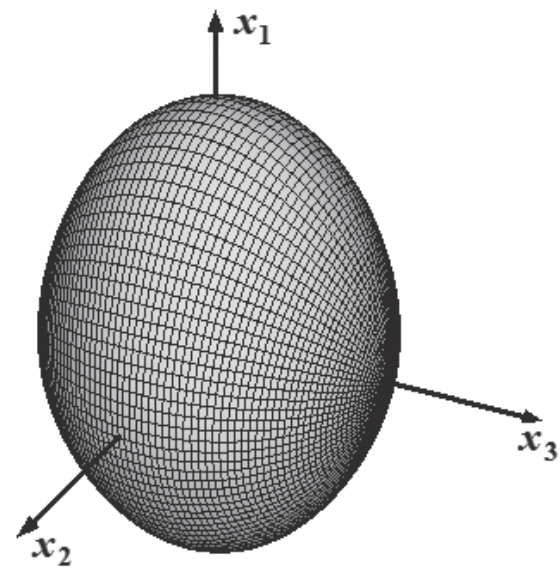

b)

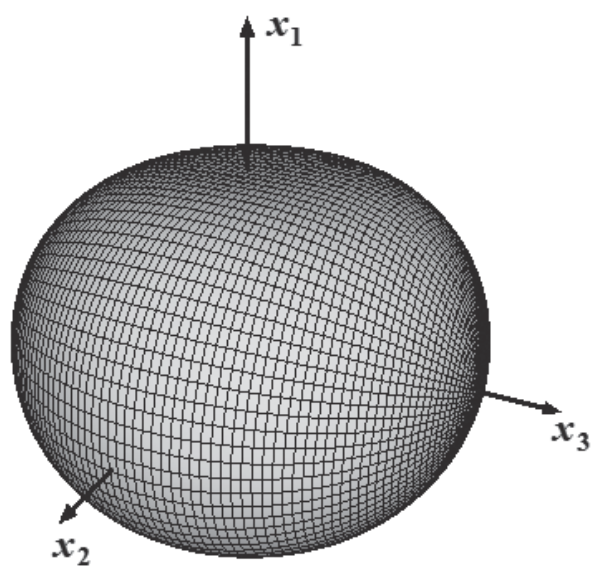

Fig. 3. Distribution of the Young of titanium depending on the direction of its examination (a); b) stored elastic energy density at uniaxial tension depending on the direction of loading

\subsection{Directions of extreme Kirchhoff modulus and extreme shear stress}

Substituting the decomposition (33) in the condition (24) after proper multiplication the nonlinear scalar equation of the condition (24) can be rewritten in terms of the components of the investigated vectors $\mathbf{x}$ and $\mathbf{y}$ in the following form:

$$
\mathrm{x}_{3} \mathrm{y}_{3}\left(\mathrm{x}_{3}^{2}-\mathrm{y}_{3}^{2}\right)=0
$$

It is clearly visible that the directions fulfilling the necessary condition for stationary value of the $g$ function are those for which either $\mathrm{x}_{3}=0$ or $\mathrm{y}_{3}=0$ or $\mathrm{x}_{3}^{2}=\mathrm{y}_{3}^{2}$, these are e.g.:

- two directions at least one of which lies in the plane perpendicular to the specified symmetry axis - these are i.e.: the direction coinciding with the specified axis and any other perpendicular to it or any two directions lying in the plane perpendicular to the specified axis,

- any two directions inclined at the same angle to the specified symmetry axis - these are i.e. two directions lying in a plane containing the specified axis, inclined equally to it or to the plane perpendicular to it.

As it was stated in case of the analysis of variation of the Kirchhoff modulus and limit shear stress in case of cubic symmetry, the above solutions are only those satisfying the necessary condition for local extremum - those proposals may be a subject of further numerical analysis.

\section{SUMMARY}

The paper was devoted to the problem of finding such directions in linear elastic anisotropic solids that certain mecha- nical properties such as longitudinal and transverse stiffness or limit uniaxial or shear stress reached their maximum or minimum value when examined in those directions. A set of necessary conditions which must be fulfilled by the component of versors determining those directions was given. Some solutions of the problem for chosen symmetries of the fourth rank tensors describing those mechanical properties of the material were indicated and analyzed. The obtained results as well as the presented methodology may be found useful in the process of optimal design of modern materials exhibiting anisotropy of their mechanical properties, such as composites or materials with internal micro- or nanostructure.

\section{References}

Berryman J.G. 2005, Bounds and self-consistent estimates for elastic constants of random polycrystals with hexagonal, trigonal, and tetragonal symmetries. J. Mech. Phys. Solids, 53, pp. 2141-2173

Burzyński W. 1928, Studium nad hipotezami wytężenia, Akademia Nauk Technicznych, Lwów 1928; see also: 2009, Selected passages from Wtodzimierz Burzyński's doctoral dissertation 'Study on material effort hypotheses", Engng. Trans., 57, 3-4, pp. 185-215

Cowin S.C., Mehrabadi M.M. 1987: On the identification of material symmetry for anisotropic elastic materials. Q. J. Mech. Appl. Math., 40, 4, pp. 451-476

Kowalczyk-Gajewska K., Ostrowska-Maciejewska J. 2009: Review on spectral decomposition of Hooke's tensor for all symmetry groups of linear elastic material. Engns. Trans., 57, 3-4, pp. 145-183

Mehrabadi M.M., Cowin S.C. 1990: Eigentensors of anisotropic elastic materials. Quart. J. Mech. Appl. Math., 43, 1, pp. 15-41

Mises R. von 1928: Mechanik der plastischen Formanderung von Kristallen. Z. Angew. Math. u. Mech., pp. 8, 161-185

Ostrowska-Maciejewska J., Rychlewski J. 2001: Generalized proper states for anisotropic elastic materials. Arch. Mech., 53, 4-5, pp. 501-518

Rychlewski J. 1984: On Hooke’s law. J. Appl. Math. Mech., 48, pp. 303-314

Yoo M.H., Fu C.L. 1998, Physical constants, deformation twinning, and microcracking of titanium aluminides, Metal. Mater. Trans. A, 29A, pp. 49-63 